\title{
PLANO PARA INTEGRAÇÃO DO BIM EM DIVERSAS DISCIPLINAS DO CURSO DE ENGENHARIA CIVIL DA UMA
}

\author{
Patrícia Escórcio $^{(1, *)}$, José Santos ${ }^{(1,2)}$ \\ (1) Universidade da Madeira, Faculdade de Ciências Exatas e da Engenharia, Departamento de \\ Engenharia Civil e Geologia, 9020-105 Funchal \\ (2) CONSTRUCT-LABEST, Faculdade de Engenharia (FEUP), Universidade do Porto \\ (*) carlota@staff.uma.pt
}

\begin{abstract}
Resumo
Após a introdução do ensino de um software BIM (Autodesk Revit) na UC de Desenho Técnico Assistido por Computador do $1^{\circ}$ ano do curso de Engenharia Civil, na Universidade da Madeira, em 2010, verificou-se que o nível de conhecimento BIM dos alunos se situa ao nível do modelador BIM e continua a ser insuficiente face às necessidades das indústrias AECO. Para uma formação mais sólida e completa na área do BIM, é importante estimular o desenvolvimento de competências de trabalho colaborativas e interdisciplinares, promover o conhecimento na área da aplicação de materiais de construção, métodos de construção, projeto de especialidades, gestão de custos e materiais, deteção de conflitos e de uma forma genérica, fomentar a utilização de outras ferramentas BIM, noutras UCs ao longo do curso.

Assim, neste trabalho é feita uma análise aos conteúdos programáticos das diferentes UCs do curso de Engenharia Civil para se verificar como e onde podem ser utilizados modelos e softwares BIM. Para tal foi necessário definir previamente um conjunto de competências necessárias para os docentes e um conjunto de competências que se pretende que os alunos atinjam. Deste modo, foi definido um plano de integração BIM para mais de uma dezena de UCs de modo a sensibilizar e motivar os respetivos docentes.
\end{abstract}

\section{Introdução}

É amplamente aceite que o Building Information Modelling (BIM) tem cada vez mais um papel fundamental no exercício de algumas atividades da indústria AECO, nomeadamente na Engenharia Civil, por permitir a gestão e a coordenação de um projeto, da sua construção e da sua manutenção [1-3].

O BIM possibilita o aumento da produtividade e da qualidade de um processo, diminuindo a duplicação de trabalho, melhorando a deteção de erros e a comunicação entre as partes [4-6]. Apesar das inúmeras vantagens para todos os intervenientes do processo, a falta de profissionais capacitados, fruto em grande parte da fraca abordagem do BIM dentro dos cursos da indústria AECO, dificulta e atrasa a inserção do BIM no mercado de trabalho $[4,5,7]$. 
Tal como tem sido constatado por vários autores [3, 8-11] é fundamental a mudança no ensino com fomento da utilização do BIM nos cursos de Engenharia Civil. Além disto, aprender BIM e utilizar BIM pode contribuir para melhorar os conhecimentos aprendidos em diversas Unidades Curriculares (UCs), já que implica a utilização e a ligação de conhecimentos de diversas UCs, nomeadamente especialidades [12].

No ensino do BIM têm sido aplicadas essencialmente duas estratégias nas universidades (Figura 1). A primeira estratégia corresponde à introdução de uma ou duas UCs específicas, geralmente no primeiro ou segundo ano, ou então nos últimos anos de curso. A segunda estratégia passa pela de utilização de modelos BIM em várias UCs ao longo do curso, normalmente como recurso e ferramenta de ensino [13].

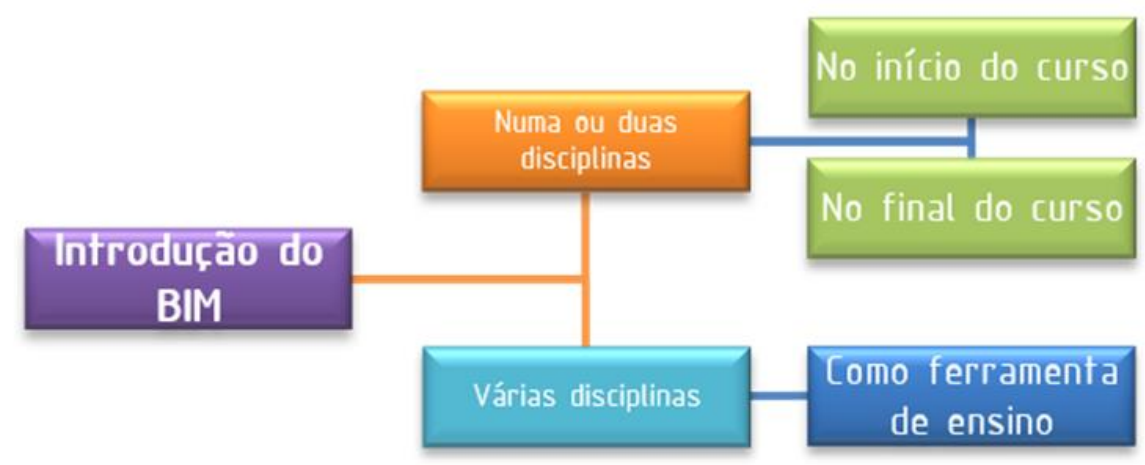

Figura 1: Estratégias de adoção BIM no ensino (adaptado de [13]).

À parte da estratégia, os objetivos são o de mudar a filosofia de pensamento e de trabalho atual fomentando a aprendizagem não só dos conceitos base da modelação BIM (BIM como modelo), mas também de como usar o BIM para comunicar diferentes tipos de informação com as diversas especialidades do projeto (BIM como metodologia de trabalho) [13].

Apesar de ser nos Estados Unidos que o ensino de BIM está mais avançado, Barison \& Santos [13] analisaram 103 universidades e concluíram que em cerca de $90 \%$ a tendência é a de introdução do BIM numa única UC, onde é ensinado normalmente um só software BIM.

Diversos autores $[13,14]$, classificam os níveis de conhecimento fruto do ensino BIM em 3 níveis, à semelhança do que é comummente aceite com os níveis de adoção BIM pelas empresas. Assim, de acordo com as estratégias de ensino adotadas podem resultar 3 níveis de conhecimento BIM: o introdutório ou modelador, o intermédio e o avançado. Na Figura 2 detalham-se estes níveis.

Contudo, como em todos os processos de mudança existem dificuldades. Os principais condicionantes à adoção do BIM no ensino, segundo diversos autores $[8,9]$ são:

- Condicionantes externos, tais como a necessidade de alterações das salas de aula, dos equipamentos, dos computadores, outro hardware e software; necessidades de suporte técnico, de manutenção e logística;

- Condicionantes associados às capacidades e preferências dos docentes;

- Condicionantes internos tais como a falta de oportunidade de usar trabalhos BIM simultaneamente em várias UCs ou o facto de os conteúdos programáticos das UCs já serem tão extensos que não permitem estar a usar o BIM em demonstrações. 

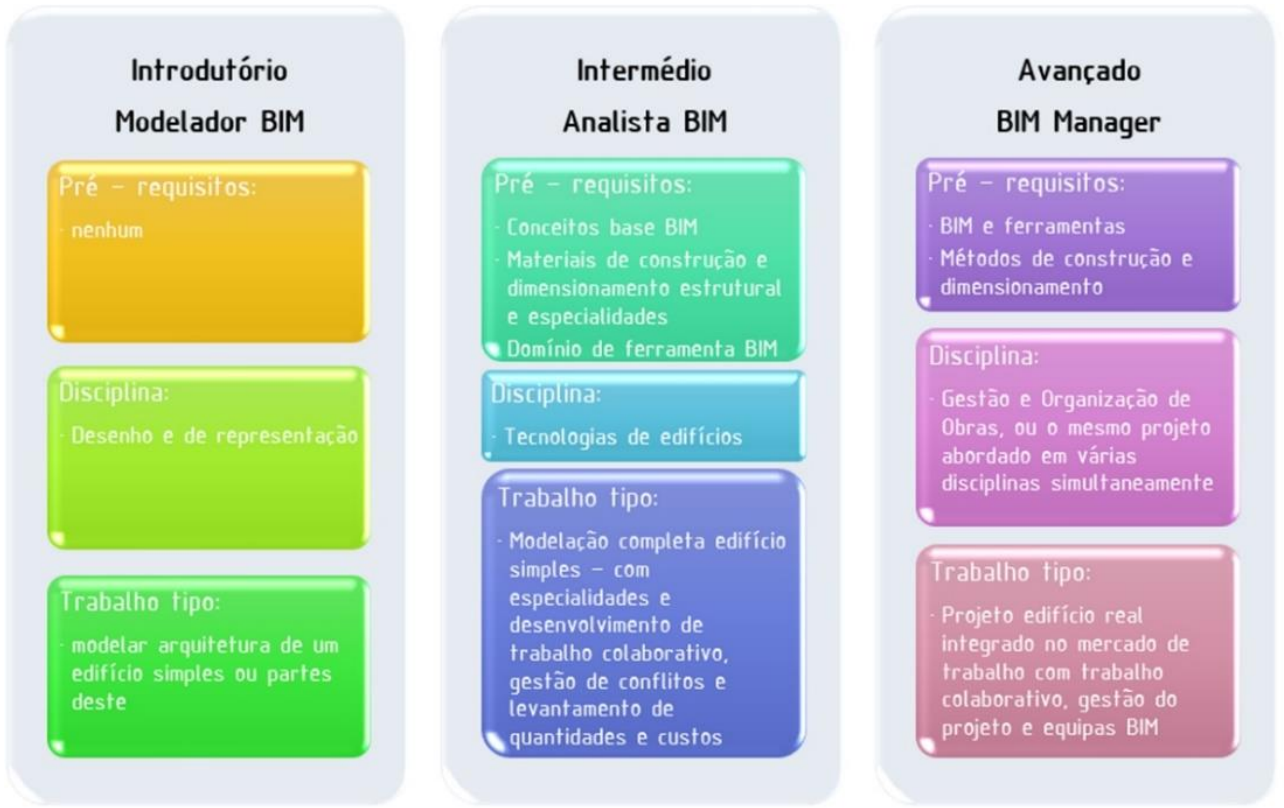

Figura 2: Níveis de conhecimento BIM (adaptado de [13]).

A integração do BIM na Universidade da Madeira (UMa) iniciou-se em 2010 com a introdução na UC de Desenho Técnico Assistido por Computador (DTAC) do ensino de um software BIM, o Autodesk REVIT (primeira estratégia anteriormente indicada).

Aquando da realização do PTBIM2018 foi feita uma reflexão sobre o grau de maturidade BIM dos alunos até essa data, bem como sobre as formas de integração do BIM noutras UCs do $1^{\circ}$ ciclo e do $2^{\circ}$ ciclo (segunda estratégia anteriormente indicada).

Relativamente ao primeiro ponto, o nível de conhecimento atingido pelos alunos que terminam esta UC corresponde ao nível básico-introdutório (execução de um modelo BIM 3D e extração de quantidades). Anualmente no final da UC têm sido realizados inquéritos aos alunos e estes mostram que, de uma forma geral, os alunos terminam a UC percebendo não só, alguns dos pontos fortes do BIM, nomeadamente, o menor tempo necessário para a execução de alguns trabalhos, e o fato de ser obter um modelo de informação do edifício e não um simples modelo 3D, como também ficam interessados em utilizar BIM futuramente.

Relativamente a este segundo ponto, verificou-se que ainda quase nada foi implementado ou modificado desde que as sugestões iniciais foram lançadas, apesar ser algo recomendável [15]. Assim, este trabalho tem como objetivo, através de uma forma mais profunda: fazer uma análise aos conteúdos programáticos das diferentes UCs para verificar como e onde podem ser utilizados modelos e softwares BIM ao longo dos cursos de $1^{\circ}$ e $2^{\circ}$ ciclo de Engenharia Civil, sugerindo um plano de integração do BIM em cada UC.

\section{Plano para a integração do BIM em diversas UCs na UMa}

Neste sentido, e tentando seguir-se uma estratégia de integração BIM que vá além da mera introdução de mais uma UC no plano de curso, neste trabalho criou-se um plano de integração BIM para diversas UCs existentes das áreas das estruturas, da hidráulica e da construção, tentando a sua adoção em demonstrações nas aulas e/ou trabalhos. 


\subsection{Metodologia}

Seguiu-se a metodologia definida na Figura 3. Partindo da avaliação da situação atual com a realização de inquéritos e do plano já definido em 2018, definiram-se aquelas que seriam as competências BIM base necessárias aos docentes das diversas UCs onde o BIM será integrado. $\mathrm{Na}$ sequência, defiram-se também as competências BIM que os alunos devem adquirir. Com estas matrizes de competências e consultando o plano de curso atual, foi feita para cada UC um plano de integração BIM detalhado, onde são elencadas as competências que o docente deve possuir para lecionar a UC, as competências que os alunos devem ter antes de frequência da UC e as que devem ter no seu término, bem como, em que momentos da UC (conteúdos programáticos) o BIM pode ser integrado quer em demonstrações nas aulas quer em trabalhos, e alguns dos softwares que podem ser utilizados.

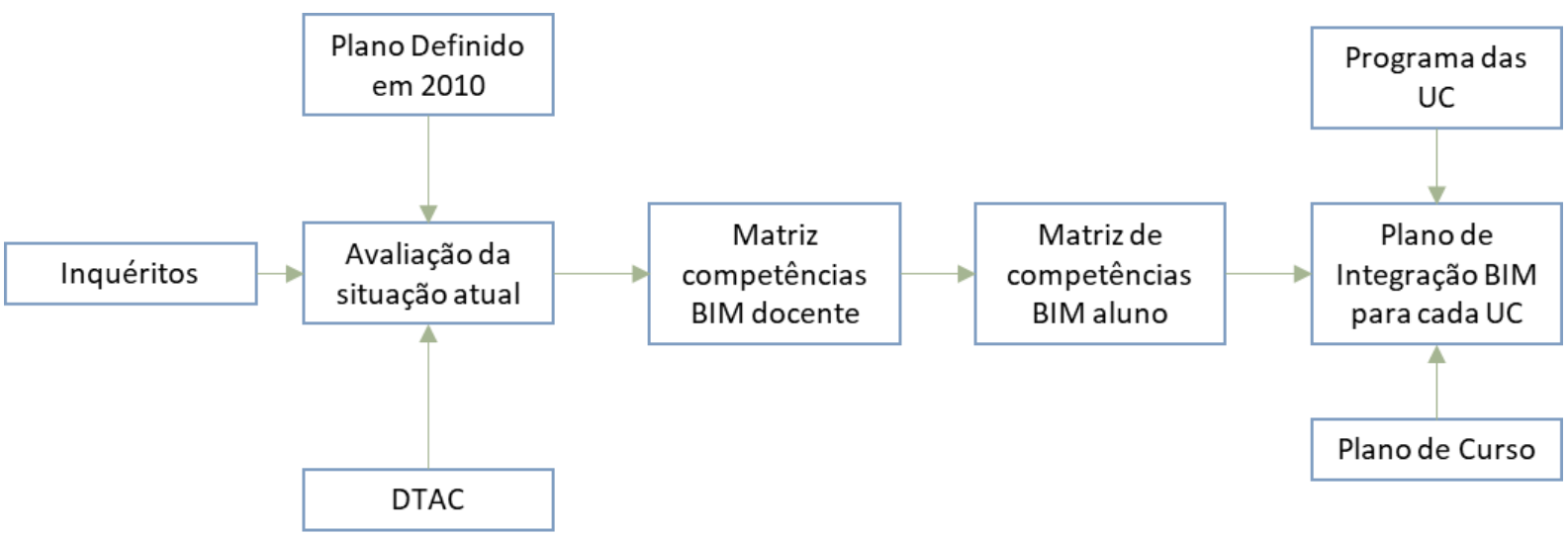

Figura 3: Metodologia

\subsection{Identificação de Competências BIM dos docentes e dos alunos e das UCs a abranger} Nas Tabelas 1 e 2, estão listadas as competências BIM que se consideraram essenciais para o docente e para o aluno, respetivamente. Definiram-se assim 13 competências BIM para os docentes e 10 para os alunos. Estas foram definidas tendo em conta a bibliografia $[6,16,17]$. Na Tabela 3 identificam-se as UCs do plano de curso atual onde se constatou que o ensino ou a utilização do BIM pode ser incluído.

\subsection{Plano de Integração BIM de cada UC}

Tendo como base as competências BIM definidas para o docente (Tabela 1) e as do aluno (Tabela 2) e os programas detalhados das UC identificadas em 2018 (Tabela 3) foi feito um plano de integração BIM para cada UC. O objetivo é o da implementação do BIM não só em contexto de trabalhos académicos, mas também no ambiente de sala de aula, como apoio em demonstrações e exemplos, ajudando os alunos a interiorizar conceitos com a visualização 3D e simulação de cenários, que é possível com um modelo BIM. 
Tabela 1: Competências BIM dos docentes

\begin{tabular}{|c|c|c|}
\hline & Competência (CD) & Descrição \\
\hline D1 & Comunicação e Gestão & $\begin{array}{c}\text { Capacidade de comunicação e gestão de novos sistemas } \\
\text { de trabalho com metodologia BIM } \\
\text { Gerir e partilhar arquivos e modelos BIM }\end{array}$ \\
\hline D2 & Facilitação & $\begin{array}{c}\text { Capacidade de modelar em BIM baseado em guias ou } \\
\text { normas ou direções especificas }\end{array}$ \\
\hline D3 & Modelação Genérica & $\begin{array}{c}\text { Capacidade de utilizar software de modelação de requisitos } \\
\text { de projeto das diferentes especialidades }\end{array}$ \\
\hline D4 & Planificação & Capacidade de planificar e desenhar \\
\hline D5 & Simulação e quantificação & $\begin{array}{c}\text { Capacidade usar um modelo BIM para gerar simulações, } \\
\text { testar hipóteses e cenários }\end{array}$ \\
\hline D6 & Operação e manutenção & $\begin{array}{l}\text { Capacidade usar um modelo BIM para gerir e manter uma } \\
\text { instalação }\end{array}$ \\
\hline D7 & Vinculação e extensão & $\begin{array}{l}\text { Capacidade de vincular um modelo BIM a outras bases de } \\
\text { dados }\end{array}$ \\
\hline D8 & Modelação personalizada & $\begin{array}{l}\text { Capacidade de produzir um modelo BIM e elementos de } \\
\text { entrega (planos, desenhos e mapas de quantidades), } \\
\text { personalizados segundo determinadas especificações }\end{array}$ \\
\hline D9 & Informática geral & Instalação e gestão de ferramentas e softwares específicos \\
\hline D10 & $\begin{array}{l}\text { Documentação e gestão do } \\
\text { modelo }\end{array}$ & $\begin{array}{c}\text { Capacidade de gerar modelos BIM de acordo com normas } \\
\text { e protocolos especificas }\end{array}$ \\
\hline D11 & Apresentação & Capacidade de gerar renders e animações 3D \\
\hline D12 & Ensino & Capacidade de produzir materiais para suporte ao ensino \\
\hline D13 & Investigação & Participação e envolvimento em estudos afetos ao BIM \\
\hline
\end{tabular}

Em algumas UCs, tais como a Hidráulica, o Betão Armado ou o Dimensionamento Estrutural, o BIM pode ser introduzido mais no contexto da modelação da estrutura e das redes, para a produção das peças desenhadas e fazendo ligação aos programas de cálculo já utilizados nessas UCs (Tabela 5). Em Organização e Gestão de Obras (Tabela 4) o contexto de utilização do BIM estará mais relacionado com a visualização e obtenção de dados de medição, geração de mapas de quantidades e faseamento construtivo (modelos 4D e 5D) e também com o papel de BIM Manager. Há ainda outras UCs em que o BIM poderia ser utilizado no âmbito de testar e comparar diversas soluções ou cenários, como exemplo a Física das Construções e a Tecnologia da Construção. 
Tabela 2: Competências BIM dos alunos

\begin{tabular}{|c|c|c|}
\hline & Competência (CA) & Descrição \\
\hline A1 & Modelação genérica & $\begin{array}{c}\text { Capacidade de utilizar software de modelação de requisitos de } \\
\text { projeto das diferentes especialidades }\end{array}$ \\
\hline A 2 & $\begin{array}{l}\text { Simulação e } \\
\text { quantificação }\end{array}$ & $\begin{array}{l}\text { Capacidade de produzir simulações e mapas de } \\
\text { quantidades/custos de acordo com determinados requisitos }\end{array}$ \\
\hline A3 & $\begin{array}{l}\text { Modelação } \\
\text { personalizada }\end{array}$ & $\begin{array}{c}\text { Capacidade de alterar um modelo BIM produzindo elementos } \\
\text { de entrega específicos }\end{array}$ \\
\hline A4 & Gestão do modelo & Capacidade de planificar, gerir e manter um modelo BIM \\
\hline A5 & Análise & $\begin{array}{l}\text { Capacidade de produzir analises, estudos num dado modelo } \\
\text { BIM }\end{array}$ \\
\hline A6 & $\begin{array}{l}\text { Gestão de projetos/ } \\
\text { trabalho colaborativo }\end{array}$ & $\begin{array}{l}\text { Capacidade de utilização e trabalho segundo a metodologia } \\
\text { BIM }\end{array}$ \\
\hline A7 & Informática Geral & $\begin{array}{l}\text { Capacidade de instalação e utilização de diversos softwares } \\
\text { BIM }\end{array}$ \\
\hline A8 & $\begin{array}{l}\text { Faseamento } \\
\text { Construtivo }\end{array}$ & $\begin{array}{l}\text { Capacidade de utilização de modelos BIM para definir } \\
\text { faseamento construtivo }\end{array}$ \\
\hline A9 & Interoperabilidade & $\begin{array}{c}\text { Capacidade de receber/enviar/realizar informação de outras } \\
\text { áreas, nomeadamente cálculo estrutural }\end{array}$ \\
\hline A10 & Vinculação e extensão & $\begin{array}{l}\text { Capacidade de vincular um modelo BIM a outras bases de } \\
\text { dados (GIS...) }\end{array}$ \\
\hline
\end{tabular}

\section{Conclusões}

A realidade atual torna fundamental o ensino do BIM nos cursos da indústria AECO, nomeadamente na Engenharia Civil. As principais motivações são a promoção e a melhoraria da aprendizagem pelos alunos, através da utilização de técnicas e métodos efetivos de comunicação e visualização digitais, assim como responder às necessidades do mercado de trabalho atual no que respeita aos processos BIM e à metodologia BIM.

A utilização de ferramentas BIM como complemento de ensino possibilita a visualização em tempo real num modelo 3D dos conteúdos da aula. Outra vantagem do uso de ferramentas BIM relaciona-se com a modelação paramétrica que permite redução de processos mecânicos e repetitivos quando se pretende mostrar várias possibilidades e simular vários cenários.

Relativamente ao ensino do BIM na UMa, apesar do plano de integração estabelecido em 2018, segundo o qual este poderia ser integrado: i) no âmbito de teses mestrado, ii) uma ou mais UCs, e iii) pela integração de softwares BIM em 12 das UCs existentes ao longo do curso, verificouse que praticamente apenas os pontos i) e ii) foram implementados. 
Tabela 3: UCs do curso de Engenharia Civil articuláveis com BIM

\begin{tabular}{|c|c|c|c|}
\hline Curso & Ano & $\mathbf{U C}$ & Áreas do BIM \\
\hline \multirow{5}{*}{$\begin{array}{l}\text { Licenciatura } \\
\left(1^{\circ} \text { ciclo }\right)\end{array}$} & 1 & $\begin{array}{c}\text { Desenho Técnico e Assistido } \\
\text { por Computador }\end{array}$ & Modelação \\
\hline & 2 & Materiais de Construção & Modelação / Visualização \\
\hline & 3 & Hidráulica & $\begin{array}{l}\text { Modelação/ interoperabilidade com } \\
\text { programas de cálculo estrutural }\end{array}$ \\
\hline & 3 & Física das Construções & Modelação / Teste de hipóteses \\
\hline & 3 & Betão Armado & $\begin{array}{l}\text { Modelação/ interoperabilidade com } \\
\text { programas de cálculo estrutural }\end{array}$ \\
\hline \multirow{7}{*}{$\begin{array}{l}\text { Mestrado } \\
\left(2^{\circ} \text { ciclo }\right)\end{array}$} & 1 & $\begin{array}{l}\text { Fundações e Estruturas de } \\
\text { Suporte }\end{array}$ & Modelação / Visualização \\
\hline & 1 & Tecnologia da Construção & Modelação / Teste de hipóteses \\
\hline & 1 & Organização e Gestão de Obras & $\begin{array}{c}\text { Visualização / Geração de orçamento / } \\
\text { Faseamento construtivo }\end{array}$ \\
\hline & 1 & $\begin{array}{c}\text { Estruturas de Betão Armado e } \\
\text { Pré-esforçado }\end{array}$ & $\begin{array}{l}\text { Modelação/ interoperabilidade com } \\
\text { programas de cálculo estrutural }\end{array}$ \\
\hline & 1 & $\begin{array}{c}\text { Reabilitação e Reforço de } \\
\text { Estruturas }\end{array}$ & $\begin{array}{l}\text { Modelação / visualização/ } \\
\text { interoperabilidade com programas de } \\
\text { cálculo estrutural }\end{array}$ \\
\hline & 2 & Dimensionamento Estrutural & $\begin{array}{l}\text { Visualização/ interoperabilidade com } \\
\text { programas de cálculo estrutural }\end{array}$ \\
\hline & 2 & Estruturas Metálicas e Mistas & $\begin{array}{l}\text { Visualização/ interoperabilidade com } \\
\text { programas de cálculo estrutural }\end{array}$ \\
\hline
\end{tabular}

Quanto à introdução em teses de mestrado importa referir que, anualmente têm sido lançados vários temas sobre a temática BIM, porém os resultados são ainda escassos e que neste caso o conhecimento BIM só é adquirido pelos alunos que optem por estes temas.

Quanto à inserção de UCs especificas de BIM além da já existente, DTAC, não é algo fácil de implementar já que exige alterações ao plano de curso e estas não são fáceis de executar, quer por questões legais quer pela necessidade de formação específica por parte dos docentes.

Quanto à utilização do BIM em diversas UCs, ainda que nenhuma dessas UCs cobrisse todas as áreas do BIM, a soma certamente proporcionaria aos alunos o conhecimento necessário para enfrentar as exigências emergentes nos mercados trabalho no que respeita ao BIM. Por isso, julga-se que a implementação desta etapa é fundamental para formar efetivamente alunos com conhecimento BIM. 
Tabela 4: Organização e Gestão de Obras

\section{Conteúdos Programáticos $(\mathrm{CP})$ :}

1. Qualificação para o Exercício da Atividade de Construção Civil e Obras Públicas.

2. Contratos Públicos.

3. Projeto. 1. Regulamentação específica. 2. Plano de gestão de resíduos. 3. Tipos de projetos. 4. Elementos de um projeto. 5. Regras de medição. 6. Estimativa de custo e orçamento da obra.

4. Equipamento de Estaleiro. 1. Modalidades de utilização de equipamento. 2. Caracterização de equipamento de utilização corrente. 3. Produção de equipamentos de estaleiro.

5. Organização e Instalação de Estaleiros. 1. Caracterização dos elementos do estaleiro. 2. Dimensionamento do estaleiro de apoio à obra

6. Metodologia de Cálculo de Custos de obras.

7. Faturação e Pagamentos. 1. Pagamentos e adiantamentos. 2. Autos de medição. 3. Revisão de preços de empreitadas.

Competências BIM do DOCENTE: BIM Manager - Informática Geral (D9); Ensino (D12);

Comunicação e gestão (D1); Facilitação (D2); Modelação genérica e especifica (D3 e D8);

Apresentação (D11); Vinculação e extensão e Deteção de incompatibilidades (D7)

\section{Competências BIM do ALUNO iniciais: Modelador BIM}

Competências BIM do ALUNO finais: Principal: Analista BIM

Informática geral (A7); Gestão do modelo (A4) Vinculação/extensão (A10); Simulação e quantificação (A2)

\begin{tabular}{llll}
\hline Correspondência & $3,4,5$ & D9, D12, D1, D3 e D8 & A7, A4, A2 \\
\cline { 2 - 4 } CP - CD - CB: & 6 & D2, D7 & A7, A2 \\
\cline { 2 - 4 } & $\mathbf{7}$ & D7, D12 & A7, A10, A2 \\
\hline
\end{tabular}

Softwares: Autodesk REVIT, ArchiCAD, Bentley ProjectWise Design Integration e ProjectWise Construction Management; Autodesk Navisworks para 4D e 5D; Solibri

A principal barreira para que esta medida continue sem ser implementada é em grande parte a falta de sensibilidade por parte dos docentes para as temáticas BIM bem como, a sua reduzida formação BIM, pelo que seria primordial investir nesta. No entanto, é fundamental que os docentes percebam que a utilização do BIM pode trazer vários benefícios, aumentando o rendimento e produtividade dos alunos, bem como o seu nível de conhecimentos.

Assim, numa nova tentativa de estimular e sensibilizar para o BIM, tendo como base as competências BIM definidas para os docentes e alunos, e os conteúdos programáticos das UC identificadas em 2018 foram feitos planos de integração do BIM nessas UCs.

Estes planos foram feitos para as UCs de Organização e Gestão de Obras, Estruturas de betão Armado e pré-esforçado, Betão Armado, Hidráulica Urbana, Física das Construções, Reabilitação e Reforço de Estruturas, Estruturas Metálicas e Mistas, Reabilitação Urbana, e Fundações e Estruturas de Suporte.

Interessa ainda referir que a implementação do BIM só será efetiva se houver uma ligação mais próxima entre o exterior e o mundo académico, de forma a que os alunos possam participar em problemas reais ganhando competências de processos colaborativos, trabalho em equipa, consultoria e dos diferentes papeis num processo BIM completo [13, 18]. Para o mercado de trabalho e para as empresas isto seria também benéfico pois ganhariam com os conhecimentos dos alunos e dos docentes em diferentes vertentes. 
Tabela 5: Estruturas de Betão Armado e Pré-esforçado

\section{Conteúdos Programáticos (CP):}

1. Introdução ao Dimensionamento de Estruturas de Betão Armado Pré-Esforçado

2. Dimensionamento e Pormenorização de Lajes de Betão Armado

3. Dimensionamento de Fundações em Betão Armado

4. Modelos de campos de tensões

Competências BIM do DOCENTE: Analista BIM - Informática Geral (D9); Ensino (D12); Comunicação e gestão (D1); Facilitação (D2); Modelação genérica e especifica (D3 e D8); Apresentação (D11); Vinculação e extensão (D7)

\section{Competências BIM do ALUNO iniciais: Modelador BIM}

Competências BIM do ALUNO finais: Principal: Modelador BIM

Informática geral (A7); Modelação genérica (A1) e personalizada (A3), Simulação e quantificação (A2), Vinculação/extensão (A10); Gestão do modelo (A4)

\begin{tabular}{llll}
\hline Correspondência & 1 & D3, D8, D12, D9 & A4, A7 \\
\cline { 2 - 4 } CP - CD - CB: & 2 & D3, D8, D1 & A1, A2 e A3 \\
\cline { 2 - 4 } & 3 & D7, D8 & A2, A3 e A4 \\
\cline { 2 - 4 } & D7, D12, D9 & A3, A4 e A10 \\
\hline
\end{tabular}

Softwares: Autodesk REVIT, ArchiCAD - uso de softwares BIM para demonstrar a colocação de armaduras. Autodesk Robot Structural analysis / SAP2000 - para cálculo dos diferentes elementos de betão Armado

\section{Referências}

[1] B. Succar, "Building Information Modelling framework: A research and delivery foundation for industry stakeholers," Automation in Construction vol. 18, pp. 357-375, 2009, doi: 10.1016/j.autcon.2008.10.003.

[2] H. Penttilä, "Describing the changes in architectural information technology to understand design complexity and free-form architectural expression," Electronic Journal of Information Technology in Construction, vol. 11, pp. 395-408, 2006.

[3] A. Abbas, Z. U. Din, and R. Farooqui, "Integration of BIM in Construction Management Education: An Overview of Pakistani Engineering Universities," Procedia Engineering, vol. 145, pp. 151-157, 2016, doi: https://doi.org/10.1016/j.proeng.2016.04.034.

[4] L. T. Knopp, P. Ferreira, and B. Costa, "Aprendizados no ensino de BIM em uma Universidade pública de interior," in A Aplicação do Conhecimento Científico na Engenharia Civil, A. Oliveira Ed.: ATENA 2019, ch. 1.

[5] P. Escórcio and J. Santos, "Integração de Software BIM na Disciplina de Desenho Técnico Assistido por Computador," presented at the $2^{\circ}$ Congresso Português de Building Information Modelling, Instituto Superior Técnico, Universidade de Lisboa, Lisboa, 2018.

[6] P. E. d. A. Basto and A. C. Lordsleem Junior, "Ensino de BIM em curso de graduação em engenharia civil em uma universidade dos EUA: estudo de caso," Ambiente Construído, vol. 16, pp. 45-61, 2016. [Online]. Available: 
http://www.scielo.br/scielo.php?script=sci_arttext\&pid=S1678-

$86212016000400045 \&$ nrm=iso.

[7] R. Sacks and R. Barak, "Teaching Building Information Modeling as an Integral Part of Freshman Year Civil Engineering Education," Journal of Professional Issues in Engineering Education and Practice, vol. 136, no. 1, pp. 30-38, 2010, doi: doi:10.1061/(ASCE)EI.1943-5541.0000003.

[8] C. M. Clevenger, M. E. Ozbek, S. Glick, and D. Porter, "Integrating BIM into Construction Management Education," 2010.

[9] H. Abdirad and C. Dossick, "BIM curriculum design in architecture, engineering, and construction education: A systematic review," Electronic Journal of Information Technology in Construction, vol. 21, pp. 250-271, 09/09 2016.

[10]R. Sacks and E. Pikas, "Building Information Modeling Education for Construction Engineering and Management. I: Industry Requirements, State of the Art, and Gap Analysis," Journal of Construction Engineering \& Management, Article vol. 139, no. 11, p. 1, 2013, doi: 10.1061/(ASCE)CO.1943-7862.0000759.

[11]R. Sacks, E. Pikas, R. Sacks, and O. Hazzan, Building Information Modeling Education for Construction Engineering and Management. II: Procedures and Implementation Case Study.

[12] J.-L. Kim, "Use of BIM for Effective Visualization Teaching Approach in Construction Education," Journal of Professional Issues in Engineering Education and Practice, vol. 138, no. 3, pp. 214-223, 2012, doi: doi:10.1061/(ASCE)EI.1943-5541.0000102.

[13]M. Barison and E. Santos, "BIM TEACHING: CURRENT INTERNATIONAL TRENDS," Gestão \& Tecnologia de Projetos, vol. 6, 02/01 2012, doi: $10.4237 / g t p . v 6 i 2.218$.

[14] M. Barison and E. Santos, "Review and Analysis of current strategies for planning a BIM curriculum," in CIB W78 2010: 27th International Conference Cairo, Egypt,, 16-18 November 2010.

[15]J. P. d. S. M. V. d. Veiga, "Do CAD para o BIM, Reflexões para o ensino da modelação em Arquitetura," Mestrado, Mestrado Integrado em Arquitetura, Universidade de Lisboa, Lisboa, 2015.

[16] J. M. V. Aparicio, "Implantacion del BIM en las competencias de la ensenanza universitaria en la escuela Professional de Ingeniería Civil de la Facultad de Arquitectura e Ingenieria Civil " Master, UNSAAC, Universidad Nacional de San Antonio Abad del Cusco, Peru, 2019.

[17]R. C. Ruschel, M. L. V. X. d. Andrade, and M. d. Morais, "O ensino de BIM no Brasil: onde estamos?," Ambiente Construído, vol. 13, pp. 151-165, 2013. [Online]. Available: http://www.scielo.br/scielo.php?script=sci_arttext\&pid=S1678$86212013000200012 \&$ nrm=iso.

[18] S. Coates, Y. Arayici, and K. Koskela, Using the Knowledge Transfer Partnership model as a method of transferring BIM and Lean process related knowledge between academia and industry: A Case Study Approach. 2018. 